\title{
Anomalous temperature dependence of the stationary Josephson tunnel current in junctions between $d$-wave superconductors
}

\author{
Alexander M. Gabovich and Alexander I. Voitenko \\ Institute of Physics, National Academy of Sciences of Ukraine, 46 Nauka Ave., Kyiv 03680, Ukraine \\ E-mail: gabovich@iop.kiev.ua; voitenko@iop.kiev.ua
}

Received March 26, 2014, published online July 21, 2014

\begin{abstract}
Stationary Josephson current $I_{c}$ between $d$-wave superconductors was calculated for superconductorinsulator-superconductor structures with various rotations of crystals relatively to each other and the junction plane. The directionality of tunneling was taken into account. It was demonstrated that the temperature, $T$, dependences of $I_{C}$ can become nonmonotonic for certain electrode orientations due to the $T$-dependent ratio between the contributions of positive and negative $I_{C}$ components. This model gives an explanation to the effect observed in junctions involving cuprates and is an alternative to the well-known scenario that makes allowance for Andreev-Saint-James zero-energy levels.
\end{abstract}

PACS: 74.20.Fg BCS theory and its development;

74.20.Rp Pairing symmetries (other than s-wave);

74.50.+r Tunneling phenomena; Josephson effects;

74.72.-h Cuprate superconductors.

Keywords: $d$-wave superconductors, cuprates, stationary Josephson current, tunnel directionality, nonmonotonic temperature dependence.

\section{Introduction}

The study of the coherent current associated with Cooper-pair tunneling between superconducting electrodes started [1,2] in the framework of tunneling-Hamiltonian approach [3]; the latter turned out to be quite satisfactory for the S-I-S junctions with wide insulating interlayers I [4,5]. Here, S denotes a superconductor. In the tunnelHamiltonian approach, when the junction transparency factor $D$ is low, the relationship between the stationary Josephson current $I_{J}$ and the difference $\varphi$ between superconducting order parameter phases on both sides of the junction is sinusoidal [1,2,6,7],

$$
I_{J}(T)=I_{C}(T) \sin \varphi,
$$

where $I_{C}$ is the critical current magnitude and $T$ is the temperature. Equation (1) holds true for isotropic $s$-wave superconductors, whereas the situation for nonconventional ones is much more involved, especially when the order parameter changes its sign over the Fermi surface (FS) $[5,8]$. This is believed to happen, e.g., in high- $T_{C}$ oxides, where $d_{x^{2}-y^{2}}$-wave superconductivity manifests itself both in phase-insensitive [9] and phase-sensitive experiments $[7,8,10]$.
It should be noted that the relation $I_{J}(\varphi)$ is no longer sinusoidal for various weak-link junctions such as constrictions or S-N-S structures ( $\mathrm{N}$ is the normal metal) even in the $s$ wave case [4,5,11-15]. Moreover, $I_{J}(\varphi)$ may change with $T$.

The influence of Andreev-Saint-James (ASJ) reflection at the S-N boundary (boundaries) [5,7,8,16-22] constitutes the physical background of possible peculiarities in the quasiparticle-current phase dependence, as well as deviations from the Ambegaokar-Baratoff behavior of the Josephson current component $[1,2]$. The latter is characterized by Eq. (1) in which $I_{C}(T)$ is a monotonic convex curve. For instance, if the boundary influence on the approaching electron is modeled by a repulsive potential $H \delta(x)$, where $x$ is the boundary position, the barrier strength is described by a dimensionless Blonder-Tinkham-Klapwijk (BTK) parameter $Z=H / \hbar v_{F}$, where $\hbar$ is Planck's constant and $v_{F}$ the electron Fermi velocity [18]. For $Z>>1$, the standard quasiparticle current-voltage characteristics of tunneling between isotropic superconductors [23] are restored. The situation with the Josephson current is more involved. Indeed, the very Ambegaokar-Baratoff expression can be interpreted as a consequence of the existing ASJ bound states, which have $\varphi$-independent energies $\pm \Delta$ in the symmetric junction. Here, $\Delta$ is the superconducting energy gap. 
In the $d$-wave superconductors, the midgap surface states at the Fermi energy level are formed at particular orientations of crystallographic axes with respect to the normal $\mathbf{n}$ to the junction plane [5,8,20,24]. For tunnel junctions, those surface states may exist at both interfaces between the superconductor $\mathrm{S}$ and the normal interlayer, and effectively influence the Josephson current $I_{J}(\varphi)$ flowing across the junction. The relation $I_{J}(\varphi)$ is known to depend strongly on the orientations of both electrodes $[8,20]$. In particular, when the orientation angles of the superconducting order parameter lobes are $\gamma=\pi / 4$ and $\gamma^{\prime}= \pm \pi / 4$ (hereafter, primed quantities are associated with the right hand side electrode), thermal depopulation of the upper ASJ surface levels leads to the enhancement of the Josephson current $I_{J}$ at low temperatures, which can be considered as the smoking gun of surface states [25-27]. A detailed account of the Josephson current flowing between $d$-wave superconductors and a comprensive lists of further references can be found in the literature [5,7,8,20,28,29].

It is worth noting that, in the simple case of normalincidence tunneling, i.e when the tunneling occurs along the vector $\mathbf{n}$ only [25,30-38], the ASJ might be neglected and the current is proportional to the simple product $\cos 2 \gamma \cos 2 \gamma^{\prime}$ [39]. The phenomenological model [39] can be considered as a useful reference point for more involved approaches and was shown to work better for diffusive electron scattering at superconducting surfaces [40].

It is important to make sure that an elegant theory proposed for normal and Josephson currents between $d$-wave superconductors with smooth boundaries $[5,7,8,20]$ remains valid for rough and dirty boundaries, which is especially crucial for thick low-transparent tunnel junctions. In particular, it was theoretically shown earlier [25,27,41-43] that the low- $T$ anomaly is rapidly suppressed as the socalled Ovchinnikov's control parameter $d / l$ increases [44]. Here, $d$ is the thickness of dirty layers coating the electrodes, and $l$ the quasiparticle mean free path. A similar model was studied by another group [45], with the same conclusions being made. If the roughness is weak, the interplay between the proximity effect at the boundary with the normal-state diffusive layer and the midgap resonance states may lead to a nonmonotonic $I_{J}(T)$ dependence [26,42,43,46-49]. Such a possibility was also obtained in the opposite limiting case $d / l>>1$ when the current $I_{J}$ itself becomes extremely small [42].

To summarize, the theory that takes into account midgap states predicts a low- $T$ anomaly in the Josephson current at some orientations of the specular reflecting $d$-wave superconducting electrodes. If the electrode surfaces are rough, the effect drastically diminishes. At the same time, the nonmonotonic behavior of $I_{J}(T)$ survives in certain cases even if the ASJ levels ultimately die out. Since one expects that cuprate superconducting order parameter or at least its dominant component has a $d$-wave symmetry, those effects should be observed for corresponding tunnel junctions.
The experimental picture seems to be rather ambiguous as well. For our purposes, it is worthwhile to point out only typical features. Relevant experiments were made for various types of junctions involving only $\mathrm{YBa}_{2} \mathrm{Cu}_{3} \mathrm{O}_{7-\delta}$ electrodes [50-56]. It turned out that a steep increase of $I_{J}(T)$ at $T \rightarrow 0$ predicted for clean junctions with the ASJ midgap states is not observed. Tunneling in $\mathrm{YBa}_{2} \mathrm{Cu}_{3} \mathrm{O}_{7-\delta}$-based edge junctions [50] is the only studied case where this phenomenon is suspected to be observed. However, its connection to the electrode orientation remained obscure. An extremely weak nonmonotonic behavior resembling the transition between the clean and rough limits in the ASJ scenario was found in grain-boundary $\mathrm{YBa}_{2} \mathrm{Cu}_{3} \mathrm{O}_{7-\delta}$ junctions $[53,56]$. Other studies of symmetric (S-I-S or S-N-S) junctions $[51,52,55]$ and nonsymmetric $\left(\mathrm{Nb}-\mathrm{Au}-\mathrm{YBa}_{2} \mathrm{Cu}_{3} \mathrm{O}_{7-\delta}\right)$ ones [54] demonstrated monotonic $I_{J}(T)$ curves for any relative orientations of the crystals involved and their orientations with respect to the junction plane.

It seems that the midgap levels inherent to $d_{x^{2}-y^{2}}$-wave superconductors either do not exist or are suppressed by the surface roughness or impurity scattering. A question arises: Can one still explain the nonmonotonic behavior of $I_{J}(T)$ for certain angular configurations, at least in S-I-S sandwiches, if the ASJ phenomenon is switched off? Below, we show that the answer is positive. The dependence $I_{J}(T)$ may be nonmonotonic owing to the well-known tunnel directionality [25,30-38], with the influence of the latter varying as the interlayer thickness changes. Our model predicts that the $I_{J}(T)$ dependences can be either monotonic or weakly nonmonotonic, without any rise of $I_{J}(T)$ at $T \rightarrow 0$. Both kinds of curves are really observed, and this qualitative agreement between the theory and the experiment may testify that (i) the midgap states in cuprate junctions are effectively wiped out by the diffusive scattering, so that the problem of Andreev scattering may be of academic interest for actual boundaries, or (ii) the superconducting order parameter includes such a subdominant component [54,57] that the nodes in the overall superconducting energy gap are absent. We note that wide enough pseudogaps (being most probably, of charge- or spindensity-wave origin) can also, in principle, occupy the node areas, thus affecting the angular and temperature dependences of Josephson current [58,59].

Our approach is similar to that developed in Ref. 31. However, the cited authors overlooked the possibility of nonmonotonic $I_{J}(T)$ behavior induced by tunnel directionality.

\section{Formulation}

In the tunnel Hamiltonian approximation [3,60,61], the stationary Josephson critical current is given by the formula $[7,62,63]$

$$
I_{C}(T)=4 e T \sum_{\mathbf{p q}}\left|\tilde{T}_{\mathbf{p q}}\right|^{2} \sum_{\omega_{n}} \mathrm{~F}^{+}\left(\mathbf{p} ; \omega_{n}\right) \mathrm{F}^{\prime}\left(\mathbf{q} ;-\omega_{n}\right) .
$$


Here, $\tilde{T}_{\mathbf{p q}}$ are the tunnel Hamiltonian matrix elements, $\mathbf{p}$ and $\mathbf{q}$ are the transferred momenta; $e>0$ is the elementary electrical charge, and $\mathrm{F}\left(\mathbf{p} ; \omega_{n}\right)$ and $\mathrm{F}^{\prime}\left(\mathbf{q} ;-\omega_{n}\right)$ are Gor'kov Green's functions for $d$-wave superconductors to the left and right, respectively, from the tunnel barrier (as was indicated above, all primed quantities are associated with the right hand side electrode). The internal summation is carried out over the discrete fermionic "frequencies" $\omega_{n}=(2 n+1) \pi T, n=0, \pm 1, \pm 2, \ldots$.

Bearing in mind an almost two-dimensional character of the FS in high- $T_{C}$ oxides, we restrict the consideration to the two-dimensional picture of tunneling, with the $c$-axes of electrodes being oriented in parallel to each other and to the junction plane. For further simplicity, only symmetric $\mathrm{S}_{d}-\mathrm{I}-\mathrm{S}_{d}$ junctions are considered, where $\mathrm{S}_{d}$ stands for the $d$-wave superconductor. In the general case (see Fig. 1), the positive lobe of the left-electrode superconducting order parameter $\Delta$ is oriented at the angle $\gamma$ with respect to the normal $\mathbf{n}$ to the junction plane. Then, the order parameter "profile" in the momentum space can be presented in the factorized form $\bar{\Delta}(T, \theta)=\Delta(T) f_{\Delta}(\theta)$, where $\Delta(T)$ is the $T$-dependent magnitude, the angular factors $f_{\Delta}(\theta)$ looks like

$$
f_{\Delta}(\theta)=\cos [2(\theta-\gamma)]
$$

and $\gamma$ is the tilt angle of the positive lobe bisectrix reckoned counterclockwise from the normal n. A similar superconductor to the right from the junction can be oriented at a different angle $\gamma^{\prime}$ with respect to the normal $\mathbf{n}$, so that

$$
\bar{\Delta}^{\prime}(T, \theta)=\Delta(T) f_{\Delta}^{\prime}(\theta)=\Delta(T) \cos \left[2\left(\theta-\gamma^{\prime}\right)\right] .
$$

Hereafter, we excluded from consideration an important ingredient of pseudogapping, which is present at almost all dopings [58,59], to study Josephson tunneling between $d$ wave superconductors just as it is.

Tunnel directionality plays one of the key roles in the proposed theory. Really, in the case when the tunnel matrix elements $T_{\mathbf{p q}}$ in formula (2) are angular-independent, the summation even over the FS section of either electrode will produce an exact zero owing to the cosine dependence (3) or (4). This is so because Green's function in Eq. (2)

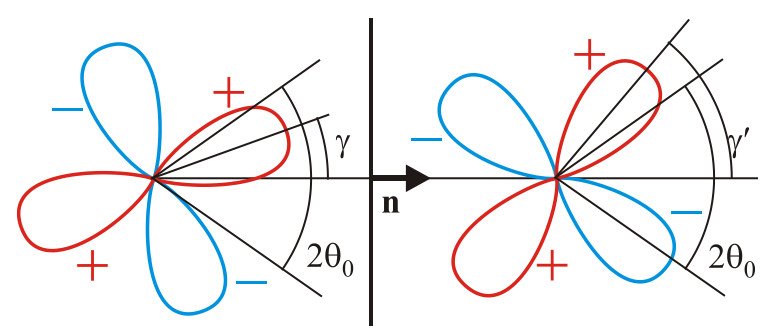

Fig. 1. (Color online) Geometry of the Josephson junction. Both electrodes are $d$-wave superconductors. The crystals are rotated at the angles $\gamma$ and $\gamma^{\prime}$, respectively, in relation to the normal $\mathbf{n}$ to the junction plane. depend on the signed superconducting order parameter. Therefore, when integrating over the FS, we include a certain FS section described by the angle $\theta$ and, inevitably, a section described by the angle $\theta \pm(\pi / 2)$, for which the corresponding values of the order parameter "profile function" $f_{\Delta}(\theta)$ - or $f_{\Delta}^{\prime}(\theta)$ - differ only by sign, so that the relevant contributions are mutually compensated. Thus, the introduction of tunnel directionality in the case of $d$-wave superconductors becomes mandatory.

While tunneling, quasiparticles and Cooper pairs demonstrate the directionality of three types. First, the quantities $\left|\mathbf{v}_{g, \text {,nd }} \cdot \mathbf{n}\right|$ and $\left|\mathbf{v}_{g, d} \cdot \mathbf{n}\right|$ appear [25,33,36], where $\mathbf{v}_{g, n d}=\nabla \xi_{\text {nd }}$ and $\mathbf{v}_{g, d}=\nabla \xi_{d}$ are the normalstate quasiparticle group velocities for proper FS sections. Those terms can be factorized into $\cos \theta$, where $\theta$ is the angle at which the pair/quasiparticle transmits through the barrier, and a factor that can be incorporated into the junction normal-state resistance $R_{N}$ [20,64-68]. Second, the tunnel matrix elements $\tilde{T}_{\mathbf{p q}}$ in (2) become momentumdependent [25,31-38,58,59,69]. In the case of isotropic superconductors (the corresponding function $f_{\Delta}(\theta)=1$ ), all gap-dependent multipliers can be taken outside the integral over $\theta$; the latter remaining hence a certain constant. This constant can also be included into the expression for $R_{N}$, which allows the directionality of this kind to be disregarded [1,2]. The third factor (the so-called coherent tunneling) inherent to the model of directional tunneling adopted here consists in that only current contributions from FS sections described by the same angle $\theta$ in both electrodes are taken into account $[31,32,70]$. In this case, the " $\Delta$-sign neutralization” in the "overlapping” FS sections takes place, and the calculation results demonstrate the experimantally observed cosinusoidal dependence of the tunnel current $I_{C}$ on the electrode misorientation angle $\gamma-\gamma^{\prime}$.

In our calculations, we made allowance for every of those factors. In particular, the barrier-associated directionality was simulated by the phenomenological function

$$
w(\theta)=\exp \left[-\left(\frac{\tan \theta}{\tan \theta_{0}}\right)^{2} \ln 2\right],
$$

so that the effective opening of relevant tunnel angles equaled $2 \theta_{0}$. The barrier transparency was normalized by the maximum value obtained for the normal tunneling with respect to the junction plane and included into the junction resistance $R_{N}$. Hence, $w(\theta=0)=1$. The multiplier $\ln 2$ in (5) was selected to ensure $w\left(\theta=\theta_{0}\right)=\frac{1}{2}$. We neglected a possible Andreev-Saint-James reflection influence on the Josephson current, because, as was indicated in Introduction, this effect is very sensitive to the interface roughness and, according to the experimental data, does not govern the Josephson flow behavior in cuprate-based junctions. 
The expression for anomalous Gor'kov Green's function for $d$-wave superconductors, which enter Eq. (2), can be found elsewhere (see, e.g., [59]). As a result, a standard for the adopted case of coherent tunneling [31,32,70] procedure $[62,63]$ leads to the following formula for the dc Josephson current across the tunnel junction:

$$
\begin{gathered}
I_{C}\left(T, \gamma, \gamma^{\prime}\right)=\frac{1}{2 e R_{N}} \times \\
\times \frac{1}{\pi} \int_{-\pi / 2}^{\pi / 2} \cos \theta w(\theta) P\left(T, \theta, \gamma, \gamma^{\prime}\right) d \theta,
\end{gathered}
$$

where [71,72]

$$
P\left(T, \theta, \gamma, \gamma^{\prime}\right)=\bar{\Delta} \bar{\Delta}^{\prime} \int_{\min \left\{\bar{\Delta}, \bar{\Delta}^{\prime}\right\}}^{\max \left\{\bar{\Delta}, \bar{\Delta}^{\prime}\right\}} \frac{\tanh \frac{x}{2 T} d x}{\sqrt{\left(x^{2}-\bar{\Delta}^{2}\right)\left(\bar{\Delta}^{\prime 2}-x^{2}\right)}} .
$$

Here, for brevity, we omitted the arguments in the dependences $\bar{\Delta}(T, \theta-\gamma)$ and $\bar{\Delta}^{\prime}\left(T, \theta-\gamma^{\prime}\right)$. Integration over $\theta$ is carried out within the interval $-\frac{\pi}{2} \leq \theta \leq \frac{\pi}{2}$, i.e. over the "FS hemicircle" turned towards the junction plane. If the directionality is so strict that $w(\theta=0)=\infty$ and zero otherwise, i.e. the Dirac delta-function, the integration over $\theta$ is elementary and the pure factors $f_{\Delta}$ and $f_{\Delta}^{\prime}$ determine the overall angular dependence, i.e. one arrives at the reference Sigrist-Rice "normal-incidence” model [73].

\section{Calculations and discussion}

The following dimensionless variables were used to present the results of calculations: the normalized temperature $t=T / T_{c}$, where $\Delta_{0}$ is $\Delta(T=0)$, and the reduced energy gap amplitude $\delta(t)=\Delta(T) / \Delta_{0}$. Besides, we normalized the Josephson current amplitude $I_{C}$ either in the conventional manner, namely $i_{c}(T)=I_{c}(T) e R_{N} / \Delta_{0}$, or as $i_{c 0}(t)=I_{c}(T) /\left|I_{c}(0)\right|$.

The results of calculations of the $i_{c}(t)$ dependence obtained in the framework of the proposed theoty for some orientations of the electrodes with respect to each other and to the junction plane are depicted in Fig. 2 by solid curves. The curve shown in panel (a) demonstrates a standard monotonic behavior within the whole temperature interval $0<T<T_{c}$. The sign of the current is governed by the orientation angles $\gamma$ and $\gamma^{\prime}$. It can be either positive or negative, the latter case coined as the $\pi$ junction $[5,8,74]$. At the same time, the curves $i_{c}(t)$ in other panels turn out nonmonotonic (panel (b)) and even sign-changing (panel (c)), although the superconducting order parameter in both electrodes varies with the temperature monotonically in the conventional manner (and identically!) in all three illustrated cases.

To analyze this strange situation, we should recall that the overall current between $d$-wave superconductors is a sum of components corresponding to tunneling connecting
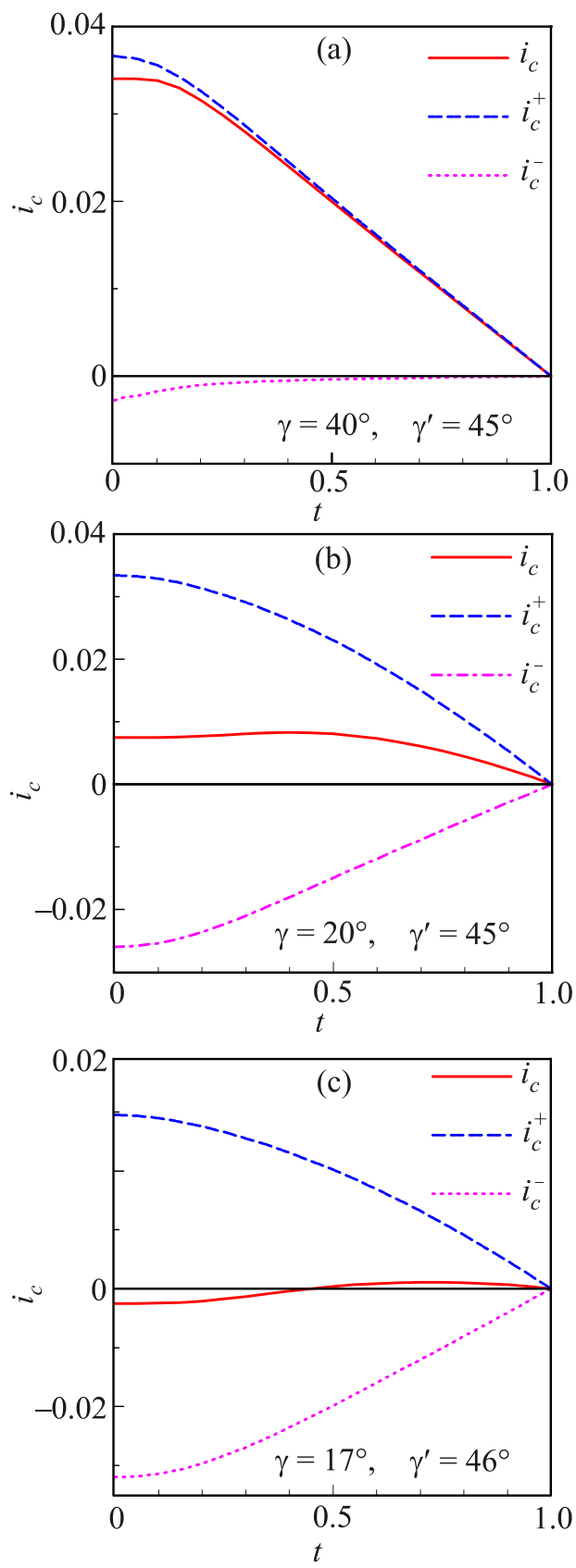

Fig. 2. (Color online) Monotonic (a), nonmonotonic (b), and sign-changing (c) dependences of the normalized stationary Josephson currents $i_{C}=I_{C} e R_{N} / \Delta_{0}$ and their positive $i_{C}^{+}$and negative $i_{C}^{-}$components on the dimensionless temperature $t=T / T_{C}$ for various orientations $\gamma$ and $\gamma^{\prime}$ of identical $d$-wave superconducting electrodes with respect to the normal $\mathbf{n}$ to the junction plane (see Fig. 1). Here $e$ is the elementary positive charge, $R_{N}$ the normal-state resistance of the tunnel junction, $\Delta_{0}$ the superconducting order parameter amplitude at $T=0$, and $T_{C}$ the critical temperature of the superconducting transition. The directionality parameter $\theta_{0}=10^{\circ}$ for all panels.

the signed order-parameter lobes that are effectively inside the angular opening $2 \theta_{0}$ and consider separately the positive, $i_{c}^{+}$, and negative, $i_{c}^{-}$, contribution to the total current $i_{c}$ (see Fig. 2). Those components are determined by the 
overlapping of co- and contra-signed, respectively, superconducting lobes in the momentun space (of course, if one angular " $\Delta$-rose" in Fig. 1 is superposed on the other). If the orientations of identical (!) electrodes are fixed, the map of overlapping sectors does not change with the temperature. Tunnel directionality assigns a specific (and also fixed!) weight for every sector with the same or the opposite $\Delta$-signs, and, at the first glance, nothing can lead to the nonmonotonic current behavior. Those speculations seem to be supported by the fact that each of the components $i_{c}^{+}(t)$ and $i_{c}^{-}(t)$ is strictly monotonic, also in all considered cases. But panels (b) and (c) testify that this is not so.

Addressing once more the basic calculation formulas (6) and (7), we arrive at the conclusion that, in addition to directionality, it is the temperature-dependent integrand factor $\tanh (x / 2 T)$ in Eq. (7) describing the temperatureinduced redistribution of fermions over the energy levels that makes this effect possible. Against the large background of each current component, the corresponding "distortion" of the temperature dependence is not conspicuous. However, tunnel directionality mixes (subtracts!) the components with different weights. When the corresponding weighted contributions become comparable, an almost complete mutual compensation of large terms makes those "distortions" observable. As a result, the situation may change drastically. In particular, for some parameter combinations, the function $i_{c}(t)$ may remain monotonic (panel (a)), but for others, it becomes nonmonotonic (panel (b)). Here, a fine interplay between the components $i_{c}^{+}$and $i_{c}^{-}$ reveals itself. Moreover, for some parameters, the mutual compensation of the components can be so effective that the total current equals zero at a certain temperature inside the interval $0<T<T_{C}$. For other $T$ 's, the current $I_{C} \neq 0$ and the same Josephson junction turns out a $\pi$-junction at low temperatures and a 0 -junction at higher ones (panel (c)), or vice versa. We would like to emphasize once more that the superconducting order parameter $\Delta$ remains a monotonic sign-preserving function of the temperature.

The very amplitude of the current is very sensitive to the mismatch between the angles $\gamma$ and $\gamma^{\prime}$, whatever the temperature, so that for one fixed crystal, the rotation of the other severely affects the outcome, similarly to what was done in well-known devices [75,76]. This can be seen from Fig. 3 where the dependence $i_{c}(0)$ versus $\gamma$ is demonstrated for $\theta_{0}=10^{\circ}$ and $\gamma^{\prime}=45^{\circ}$. Indeed, at $\gamma=0^{\circ}$, the positive and negative current components are mutually compensated, whereas for another extreme symmetric configuration, $i_{c}(0)$ reaches the maximum value.

The following question arises: What are the conditions for the thermally nonmonotonic behavior of the Josephson tunnel current to be observed? As was indicated above while analyzing Fig. 2, the monotonic behavior takes place when the superconducting lobes in both electrodes strongly overlap. In this case, large overlapped FS areas provide large current contributions of the same sign, dominating in

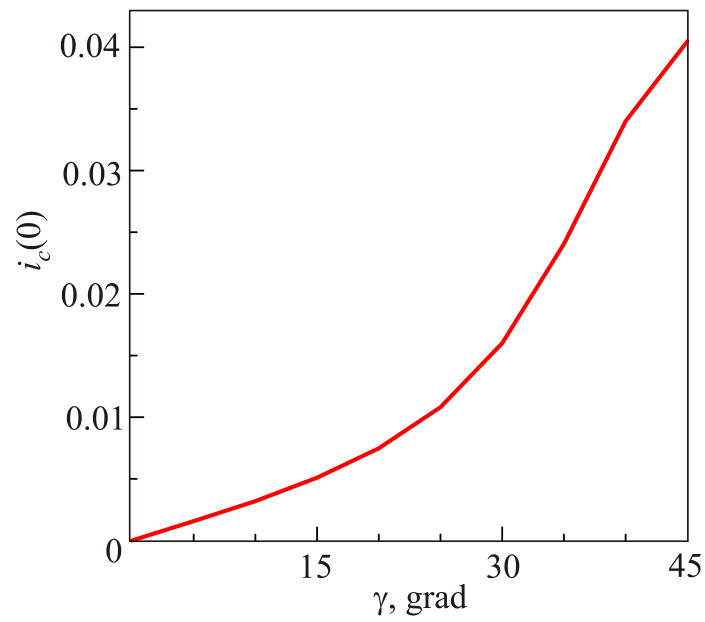

Fig. 3. (Color online) Dependence of the value $i_{C}(0)$ on the angle $\gamma$ at $\theta_{0}=10^{\circ}$ and $\gamma^{\prime}=45^{\circ}$.

the current and determining its monotonic behavior. As is shown in Fig. 2(b), the electrode orientation mismatch $\gamma-\gamma^{\prime}$ must be rather large for the effect concerned to be detectable. Nevertheless, the results of calculations depicted in Fig. 4 testify that this is feasible. Moreover, the nonstandard behavior of the current can be obtained by varying any of the parameters $\left(\gamma, \gamma^{\prime}, \theta_{0}\right)$. Of course, the values of the other parameters must be correspondingly chosen. The conditions are much stricter (the intervals of parameter change are much narrower) if we would like to observe the temperature-governed transformation of a $\pi$-junction to 0 one or vice versa. It is of importance that neither of the parameters $\left(\gamma, \gamma^{\prime}, \theta_{0}\right)$ is associated with the electrode material, but only with the junction geometry. Our theory perdicts that, for the same $d$-wave superconductor used as electrodes, we can obtain both monotonic and non monotonic $I_{C}(T)$ dependences. In the latter case, there may be a $T$-driven transition between 0 - and $\pi$-branches, or the absence of this transition when the 0 (or $\pi$ )-branch persists in the whole range $0<T<T_{C}$. The first situation was actually observed in $\mathrm{YBa}_{2} \mathrm{Cu}_{3} \mathrm{O}_{7-\delta}$ grain-boundary Josephson junctions [53]. The second possibility was most probably realized in $\mathrm{YBa}_{2} \mathrm{Cu}_{3} \mathrm{O}_{7-\delta}$ bicrystal grainboundary junctions [56].

Thus, directionality together with the thermal redistribution of quasiparticles can explain the nonmonotonic character of the $I_{C}(T)$ curve without invoking the ASJ effect. It is instructive to analyze in more detail (cf. Fig. 4(b)) how the reduction of directionality alone affects the current behavior found above. In Figs. 5(a) and (b), the dependences $i_{c 0}(t)$ are displayed for $\theta_{0}=10^{\circ}$ and $30^{\circ}$, respectively, whereas in panel (c), the dependences $i_{c}(t)$ for $\theta_{0}=90^{\circ}$ are plotted. The latter was done for two reasons. First, the corresponding $i_{c 0}(t)$ dependences are almost indistinguishible from each other (cf. the convergence of the curves when changing from the case $\theta_{0}=10^{\circ}$ to the $\theta_{0}=30^{\circ}$ one). Second, the plots $i_{c}(t)$ describe the influ- 

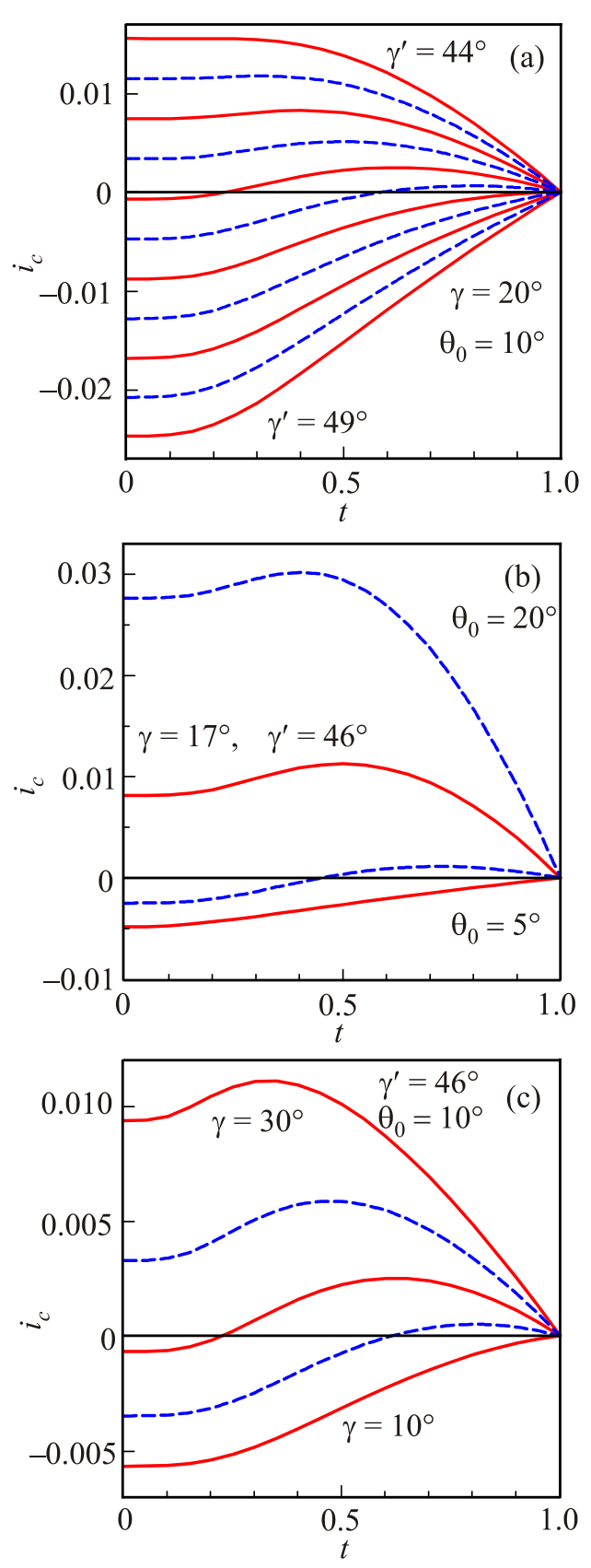

Fig. 4. (Color online) Dependences $i_{C}(t)$ illustrating the realization of any of three possible $i_{C}(t)$ patterns by varying the corresponding parameters for certain configurations.

ence of the relevant parameters on the current magnitude. It is almost the same in all analyzed cases for various $\theta_{0}$. The variation of the normalization quantity $I_{c}(0)$ can be understood from Fig. 3. Anyway, one sees that the increase of the angle $\theta_{0}$ results in a gradual disappearance of the non-monotonic current behavior. When the directionality associated with the barrier transmission coefficient (5) does not exist $\left(\theta_{0}=90^{\circ}\right)$, all the curves $I_{C}(T)$ are monotonic and smooth as is shown in panel (c).

Hence, nonmonotonic dependences should be better observed for thick enough insulator interlayers, for which tunnel directionality that ASJ resonant states do not play any
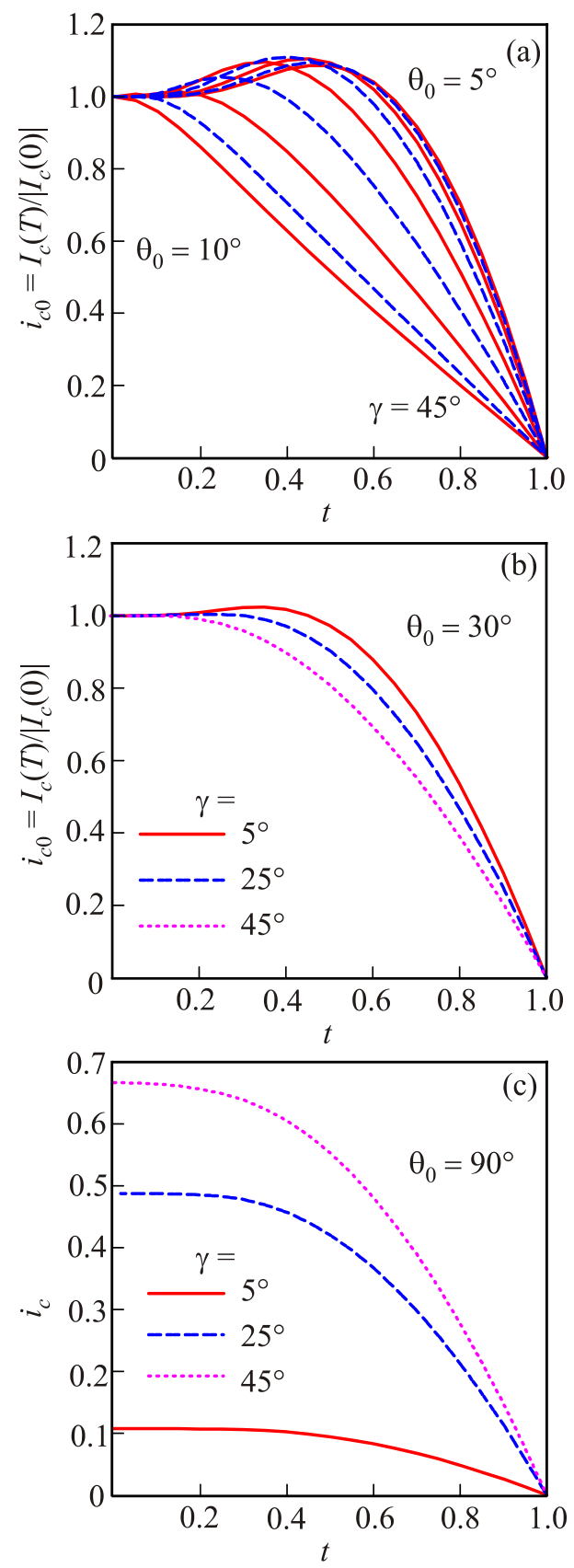

Fig. 5. (Color online) Dependences of the reduced Josephson current $i_{c 0}$ (a) and (b) and the normailized current $i_{C}$ (c) on $t$ for various $\gamma$ and $\theta_{0}$, and at the fixed $\gamma^{\prime}=45^{\circ}$. See explanations in the text.

significant role in the experiments discussed abovlity is stronger. It seems that ASJ resonant states do not play any significant role in the experiments discussed above due to the surface roughness [25,27,41-43], which eliminates the corresponding energy levels predicted for Josephson junctions made of $d$-wave superconductors [5,7,8,20,28,29]. Another, mandatory, effect predicted in the framework of the ASJ paradigm-namely, the steep increase of $I_{C}(T)$ at $T \rightarrow 0$ - also seems not to be actual for experiments with cuprate tunnel junctions. We showed that directionality does not lead to the low- $T$ anomaly as well. 
As a result, the following remark may be useful while planning the experiment and interpreting its results. For the ASJ effect to be detected, the junction should be formed between rather smooth atomic surfaces of superconducting crystals. In the case of high- $T_{c}$ oxides with the $d_{x^{2}-y^{2}}$-wave symmetry, these are presumably planes that are normal to the orientations of superconducting lobes (see Fig. 1), i.e. to the cases when $\left(\gamma, \gamma^{\prime}\right) \approx 0$ or $\pm \pi / 2$. On the other hand, our theory predicts the non-monotonic behavior of $I_{C}$ in the case when one of the electrodes is oriented at an angle close to $\pm \pi / 4$ and plays the role of differential detector.

\section{Conclusions}

Calculations of the stationary Josephson current $I_{c}(T)$ between $d$-wave superconductors were carried out taking into account tunnel directionality but ignoring possible zeroenergy ASJ midgap states. The theory enables an important feature observed in experiments involving cuprates to be explained. Specifically, the temperature dependence of $I_{C}$ can become nonmonotonic for high enough directionality and at certain crystal lattice orientations with respect to the junction plane. The peculiar behavior emerges because of a substantial compensation between the positive and negative components of $I_{C}(T)$ driven by tunnel directionality. A similar result was obtained some time ago in a scenario when ASJ energy levels in the sandwich play a significant role. At the same time, our model does not lead to the low- $T$ anomaly predicted when the ASJ effect is made allowance for. Relevant measurements also did not reveal such an anomaly. Therefore, even if the ASJ effect is suppressed by impurity- or roughness-governed scattering (this seems to be the case for cuprates), the nonmonotonic behavior still can exist due to another reason suggested here.

The work was partially supported by the Project No. 8 of the 2012-2014 Scientific Cooperation Agreement between Poland and Ukraine. The authors are grateful to Alexander Kasatkin (Institute of Metal Physics, Kyiv) for useful remarks.

1. V. Ambegaokar and A. Baratoff, Phys. Rev. Lett. 10, 486 (1963).

2. V. Ambegaokar and A. Baratoff, Phys. Rev. Lett. 11, 104 (1963).

3. J. Bardeen, Phys. Rev. Lett. 6, 57 (1961).

4. W. Haberkorn, H. Knauer, and J. Richter, Phys. Status Solidi A 47, K161 (1978).

5. A.A. Golubov, M.Yu. Kupriyanov, and E. Il'ichev, Rev. Mod. Phys. 76, 411 (2004).

6. B.D. Josephson, Phys. Lett. 1, 251 (1962).

7. F. Tafuri and J.R. Kirtley, Rep. Prog. Phys. 68, 2573 (2005).

8. T. Löfwander, V.S. Shumeiko, and G. Wendin, Supercond. Sci. Technol. 14, R53 (2001).
9. J.F. Annett, N.D. Goldenfeld, and A.J. Leggett, in: Physical Properties of High Temperature Superconductors V.D.M. Ginsberg (ed.), World Scientific, River Ridge, NJ (1996), p. 375.

10. C.C. Tsuei and J.R. Kirtley, in: Superconductivity, Vol. 2: Novel Superconductors, K.H. Bennemann and J.B. Ketterson (eds.), Springer Verlag, Berlin (2008), p. 869.

11. I.O. Kulik and A.N. Omelyanchuk, Pis'ma Zh. Éksp. Teor. Fiz. 21, 216 (1975).

12. I.O. Kulik and A.N. Omel'yanchuk, Fiz. Nizk. Temp. 3, 945 (1977) [Sov. J. Low Temp. Phys. 3, 840 (1977)].

13. I.O. Kulik and A.N. Omel'yanchuk, Fiz. Nizk. Temp. 4, 296 (1978) [Sov. J. Low Temp. Phys. 4, 142 (1978)].

14. K.K. Likharev, Rev. Mod. Phys. 51, 101 (1979).

15. M.Yu. Kuprianov and V.F. Lukichev, Zh. Éksp. Teor. Fiz. 94, 139 (1988).

16. A.F. Andreev, Zh. Eksp. Teor. Fiz. 46, 1823 (1964).

17. D. Saint-James, J. Phys. (Paris) 25, 899 (1964).

18. G.E. Blonder, M. Tinkham, and T.M. Klapwijk, Phys. Rev. B 25, 4515 (1982).

19. P.A.M. Benistant, A.P. van Gelder, H. van Kempen, and P. Wyder, Phys. Rev. B 32, 3351 (1985).

20. S. Kashiwaya and Y. Tanaka, Rep. Prog. Phys. 63, 1641 (2000).

21. G. Deutscher, Rev. Mod. Phys. 77, 109 (2005).

22. D. Daghero, M. Tortello, P. Pecchio, V.A. Stepanov, and R.S. Gonnelli, Fiz. Nizk. Temp. 39, 261 (2013) [Low Temp. Phys. 39, 189 (2013)].

23. A.I. Larkin and Yu.N. Ovchinnikov, Zh. Eksp. Teor. Fiz. 51, 1535 (1966).

24. C.-R. Hu, Phys. Rev. Lett. 72, 1526 (1994).

25. Yu.S. Barash, H. Burkhardt, and D. Rainer, Phys. Rev. Lett. 77, 4070 (1996).

26. Y. Tanaka and S. Kashiwaya, Phys. Rev. B 53, 11957 (1996).

27. R.A. Riedel and P.F. Bagwell, Phys. Rev. B 57, 6084 (1998).

28. F. Tafuri, J.R. Kirtley, F. Lombardi, P.G. Medaglia, P. Orgiani, and G. Balestrino, Fiz. Nizk. Temp. 30, 785 (2004) [Low Temp. Phys. 30, 591 (2004)].

29. F. Tafuri, D. Massarotti, L. Galletti, D. Stornaiuolo, D. Montemurro, L. Longobardi, P. Lucignano, G. Rotoli, G.P. Pepe, A. Tagliacozzo, and F. Lombardi, J. Supercond. 26, 21 (2013).

30. E.L. Wolf, Principles of Electron Tunneling Spectroscopy, Oxford University Press, New York (1985).

31. C. Bruder, A. van Otterlo, and G.T. Zimanyi, Phys. Rev. B 51, 12904 (1995).

32. M. Ledvij and R.A. Klemm, Phys. Rev. B 51, 3269 (1995).

33. Yu.S. Barash, A.V. Galaktionov, and A.D. Zaikin, Phys. Rev. B 52, 665 (1995).

34. K. Kouznetsov and L. Coffey, Phys. Rev. B 54, 3617 (1996).

35. Y-m. Nie and L. Coffey, Phys. Rev. B 57, 3116 (1998).

36. Y-m. Nie and L. Coffey, Phys. Rev. B 59, 11982 (1999).

37. Yu.M. Shukrinov, A. Namiranian, and A. Najafi, Fiz. Nizk. Temp. 27, 15 (2001) [Low Temp. Phys. 27, 10 (2001)].

38. A. Sharoni, G. Leibovitch, A. Kohen, R. Beck, G. Deutscher, G. Koren, and O. Millo, Europhys. Lett. 62, 883 (2003).

39. M. Sigrist and T.M. Rice, Rev. Mod. Phys. 67, 503 (1995). 
40. T. Yokoyama, Y. Sawa, Y. Tanaka, and A.A. Golubov, Phys. Rev. B 75, 020502 (2007).

41. A. Poenicke, Yu.S. Barash, C. Bruder, and V. Istyukov, Phys. Rev. B 59, 7102 (1999).

42. A.A. Golubov and M.Yu. Kupriyanov, Pis'ma Zh. Eksp. Teor. Fiz. 69, 242 (1999).

43. T. Lück, P. Schwab, U. Eckern, and A. Shelankov, Phys. Rev. B 68, 174524 (2003).

44. Yu.N. Ovchinnikov, Zh. Eksp. Teor. Fiz. 56, 1590 (1969).

45. T. Yokoyama, Y. Sawa, Y. Tanaka, A.A. Golubov, A. Maeda, and A. Fujimaki, Phys. Rev. B 76, 052508 (2007).

46. S-K. Yip, J. Low Temp. Phys. 109, 547 (1997).

47. Y. Tanaka and S. Kashiwaya, Phys. Rev. B 56, 892 (1997).

48. T. Yokoyama, Y. Tanaka, A.A. Golubov, and Y. Asano, Phys. Rev. B 73, 140504 (2006).

49. T. Yokoyama, Y. Tanaka, and A.A. Golubov, Phys. Rev. B 75, 094514 (2007).

50. A.W. Kleinsasser and K.A. Delin, Appl. Phys. Lett. 66, 102 (1995).

51. G. Koren, E. Polturak, N. Levy, and G. Deutscher, Phys. Rev. B 61, 3734 (2000).

52. H. Arie, K. Yasuda, H. Kobayashi, I. Iguchi, Y. Tanaka, and S. Kashiwaya, Phys. Rev. B 62, 11864 (2000).

53. E. Il'ichev, M. Grajcar, R. Hlubina, R.P.J. IJsselsteijn, H.E. Hoenig, H-G. Meyer, A. Golubov, M.H.S. Amin, A.M. Zagoskin, A.N. Omelyanchouk, and M.Yu. Kupriyanov, Phys. Rev. Lett. 86, 5369 (2001).

54. G.A. Ovsyannikov, P.V. Komissinski, E. Il'ichev, Y.V. Kislinski, and Z.G. Ivanov, IEEE Trans. Appl. Supercond. 13, 881 (2003).

55. J. Yoshida, H. Katsuno, K. Nakayama, and T. Nagano, Phys. Rev. B 70, 054511 (2004).

56. G. Testa, E. Sarnelli, A. Monaco, E. Esposito, M. Ejrnaes, D-J. Kang, S.H. Mennema, E.J. Tarte, and M. G. Blamire, Phys. Rev. B 71, 134520 (2005).

57. Š. Kos, Phys. Rev. B 63, 214506 (2001).
58. A.M. Gabovich and A.I. Voitenko, Fiz. Nizk. Temp. 38, 414 (2012) [Low Temp. Phys. 38, 326 (2012)].

59. A.M. Gabovich and A.I. Voitenko, Fiz. Nizk. Temp. 39, 301 (2013) [Low Temp. Phys. 39, 232 (2013)].

60. W.A. Harrison, Phys. Rev. 123, 85 (1961).

61. M.H. Cohen, L.M. Falicov, and J.C. Phillips, Phys. Rev. Lett. 8, 316 (1962).

62. I.O. Kulik and I.K. Yanson, Josephson Effect in Superconducting Tunnel Structures, Israel Program for Scientific Translation, Jerusalem (1972).

63. A. Barone and G. Paterno, The Physics and Applications of the Josephson Effect, John Wiley and Sons, New York (1982).

64. Y. Tanaka, Phys. Rev. Lett. 72, 3871 (1994).

65. J.H. Xu, J.L. Shen, J.H. Miller, Jr, and C.S. Ting, Phys. Rev. Lett. 73, 2492 (1994).

66. Yu.S. Barash, A.V. Galaktionov, and A.D. Zaikin, Phys. Rev. Lett. 75, 1676 (1995).

67. J.H. Xu, J.L. Shen, J.H. Miller, Jr, and C.S. Ting, Phys. Rev. Lett. 75, 1677 (1995).

68. Yu.S. Barash and A.A. Svidzinskii, Zh. Eksp. Teor. Fiz. 111, 1120 (1997).

69. A.M. Gabovich, M.S. Li, H. Szymczak, and A.I. Voitenko, Phys. Rev. B 87, 104503 (2013).

70. R.A. Klemm, Philos. Mag. 85, 801 (2005).

71. A.E. Gorbonosov and I.O. Kulik, Fiz. Met. Metalloved. 23, 803 (1967).

72. A.M. Gabovich, D.P. Moiseev, A.S. Shpigel, and A.I. Voitenko, Phys. Status Solidi B 161, 293 (1990).

73. M. Sigrist and T.M. Rice, J. Phys. Soc. Jpn. 61, 4283 (1992).

74. H. Hilgenkamp, Supercond. Sci. Technol. 21, 034011 (2008).

75. H.J.H. Smilde, A.A. Golubov, A. Ariando, G. Rijnders, J.M. Dekkers, S. Harkema, D.H.A. Blank, H. Rogalla, and H. Hilgenkamp, Phys. Rev. Lett. 95, 257001 (2005).

76. J.R. Kirtley, C.C. Tsuei, A. Ariando, C.J.M. Verwijs, S. Harkema, and H. Hilgenkamp, Nature Phys. 2, 190 (2006). 